\title{
Fracture of fusion mass after hardware removal in patients with high sagittal imbalance
}

\author{
Cara L. Sedney, MD, MA, ${ }^{1}$ Scott D. Daffner, MD, ${ }^{2}$ Jared J. Stefanko, DO, ${ }^{3}$ \\ Hesham Abdelfattah, MD, ${ }^{2}$ Sanford E. Emery, MD, MBA, ${ }^{2}$ and John C. France, MD ${ }^{2}$ \\ Departments of ${ }^{1}$ Neurosurgery and ${ }^{2}$ Orthopaedics, West Virginia University, Morgantown, West Virginia; and ${ }^{3}$ Spectrum \\ Orthopaedics, Inc., Canton, Ohio
}

\begin{abstract}
OBJECT As spinal fusions become more common and more complex, so do the sequelae of these procedures, some of which remain poorly understood. The authors report on a series of patients who underwent removal of hardware after CT-proven solid fusion, confirmed by intraoperative findings. These patients later developed a spontaneous fracture of the fusion mass that was not associated with trauma. A series of such patients has not previously been described in the literature.
\end{abstract}

METHODS An unfunded, retrospective review of the surgical logs of 3 fellowship-trained spine surgeons yielded 7 patients who suffered a fracture of a fusion mass after hardware removal. Adult patients from the West Virginia University Department of Orthopaedics who underwent hardware removal in the setting of adjacent-segment disease (ASD), and subsequently experienced fracture of the fusion mass through the uninstrumented segment, were studied. The medical records and radiological studies of these patients were examined for patient demographics and comorbidities, initial indication for surgery, total number of surgeries, timeline of fracture occurrence, risk factors for fracture, as well as sagittal imbalance.

RESULTS All 7 patients underwent hardware removal in conjunction with an extension of fusion for ASD. All had CTproven solid fusion of their previously fused segments, which was confirmed intraoperatively. All patients had previously undergone multiple operations for a variety of indications, 4 patients were smokers, and 3 patients had osteoporosis. Spontaneous fracture of the fusion mass occurred in all patients and was not due to trauma. These fractures occurred 4 months to 4 years after hardware removal. All patients had significant sagittal imbalance of $13-15 \mathrm{~cm}$. The fracture level was L-5 in 6 of the 7 patients, which was the first uninstrumented level caudal to the newly placed hardware in all 6 of these patients. Six patients underwent surgery due to this fracture.

CONCLUSIONS The authors present a case series of 7 patients who underwent surgery for ASD after a remote fusion. These patients later developed a fracture of the fusion mass after hardware removal from their previously successfully fused segment. All patients had a high sagittal imbalance and had previously undergone multiple spinal operations. The development of a spontaneous fracture of the fusion mass may be related to sagittal imbalance. Consideration should be given to reimplanting hardware for these patients, even across good fusions, to prevent spontaneous fracture of these areas if the sagittal imbalance is not corrected.

http://thejns.org/doi/abs/10.3171/2015.7.SPINE15153

KEY WORDS spinal fusion; adjacent-segment disease; sagittal balance; fracture; back pain; complication; technique

$\Lambda$ s spinal fusions become more common and more complex, so do the sequelae of these procedures, which are sometimes poorly understood. We report on a series of patients who underwent removal of hardware after CT-proven solid fusion, further confirmed by intraoperative findings. These patients later developed a spontaneous fracture of the fusion mass that was not associated with trauma.

Previous reports of fracture through fused levels have included 1 or 2 patients and represent a variety of patient populations. Ha and colleagues presented 2 separate case reports, one in which a compression fracture ${ }^{4}$ and another

ABBREVIATIONS ASD = adjacent-segment disease; $\mathrm{BMP}=$ bone morphogenetic protein; $\mathrm{DISH}=$ diffuse idiopathic skeletal hyperostosis; $\mathrm{LL}=$ lumbar lordosis; $\mathrm{PI}=$ pelvic incidence; $S V A=$ sagittal vertical axis.

SUBMITTED February 3, 2015. ACCEPTED July 29, 2015.

INCLUDE WHEN CITING Published online December 18, 2015; DOI: 10.3171/2015.7.SPINE15153. 
in which bilateral pedicle stress fractures ${ }^{3}$ were sustained after removal of hardware and extension of fusion. Ito and colleagues have reported on 3 patients who sustained a fracture through an anterior strut graft after spondylectomy. ${ }^{5}$ Finally, Waelchli and colleagues reported on 2 patients who experienced a refracturing of their thoracolumbar fracture after hardware removal. ${ }^{12}$ To our knowledge, ours is the first single series of such patients, enabling closer examination of the possible risk factors for this serious complication following implant removal.

\section{Methods}

After institutional review board approval, a retrospective chart review was conducted to assess the incidence, sequelae, and risk factors for spontaneous fusion mass fracture after hardware removal. There was no funding for this project. The operative case logs of the 3 fellowship-trained spine surgeons at an academic institution from 2008 to 2012 were investigated. All patients who underwent removal of hardware from the lumbar spine were initially identified. Patients who demonstrated a successful fusion via $\mathrm{CT}$, with further documented solid fusion following intraoperative exploration of the fusion mass, and who subsequently developed a spontaneous (atraumatic) CT-proven fracture through the fusion mass were included. Patients who experienced fusion mass fracture due to trauma were excluded. Patients who had hardware removed and did not sustain a fracture were used as a control group.

For those patients experiencing fracture, medical records were examined. Age, sex, indication, date of index procedure, total number of surgeries, number of fused levels, indication for hardware removal, spinal level of fracture, timeline of fracture occurrence, smoking history, diagnosis of osteoporosis, diffuse idiopathic skeletal hyperostosis (DISH), and ankylosing spondylitis were recorded. Imaging was reviewed to confirm initial solid fusion, presence of fracture, and location of fracture. Sagittal balance was assessed and sagittal vertical axis (SVA), lumbar lordosis (LL), and pelvic incidence (PI) were calculated using 36-inch, standing scoliosis radiographs.

\section{Results}

During the time period under review, 747 thoracolumbar fusions, 117 hardware removals, and 71 explorations of fusion were performed. Patients who had their hardware replaced in the same surgery or same hospitalization, those with unilateral hardware removal, and those with hardware removal after internal fixation only rather than bone fusion were excluded from further analysis. Fortythree patients were included for analysis. Seven patients (16\%) had CT- and intraoperatively proven solid fusion, subsequent hardware removal, and a delayed spontaneous fracture of a previously fused segment from which the hardware had been removed (Table 1). Thirty-six patients underwent hardware removal but did not experience a fracture and were used as a control group.

\section{Patient Description}

Five of the 7 patients experiencing fracture were female, and all were over 50 years of age. All patients had previously undergone multiple spinal surgeries (ranging from 3 to 8), with the index procedure performed for degenerative disease (including stenosis and spondylolisthesis) in 4 patients, scoliosis in 1 patient, discitis in 1 patient, and unknown in 1 patient. The index procedures were all remote, with the earliest occurring more than 30 years before presentation to our institution. Fusion materials used in the index procedures varied and included autologous graft, allograft, and bone morphogenetic protein (BMP). Four patients were smokers. Three patients had confirmed osteoporosis. The other 4 did not have a formal diagnosis of osteoporosis, but the diagnosis had not been excluded with the diagnostic tests available for our review. No patient had a diagnosis of ankylosing spondylitis or DISH.

\section{Surgical History}

All 7 patients had undergone removal of previously placed hardware during a surgery for which their fusion was being extended due to symptomatic adjacent-segment disease (ASD). One patient had adjacent segment degeneration but did not want an extension of fusion; for this patient, the hardware was removed and replaced only at a level of nonunion within the previous fusion. A discussion of performing a larger procedure for correction of overall sagittal balance was held with these patients, but in most cases the patients were believed to not be medically stable or refused the larger procedure in favor of a smaller procedure. Two patients had already received a pedicle subtraction osteotomy as part of a previous procedure to

TABLE 1. Characteristics of the 7 patients who suffered fracture of fusion mass after hardware removal

\begin{tabular}{|c|c|c|c|c|c|c|c|c|c|}
\hline $\begin{array}{l}\text { Case } \\
\text { No. }\end{array}$ & $\begin{array}{l}\text { Age (yrs), } \\
\text { Sex }\end{array}$ & Initial Pathology & $\begin{array}{l}\text { Previous } \\
\text { Fusion }\end{array}$ & Extended Levels & $\begin{array}{c}\text { Fracture } \\
\text { Level }\end{array}$ & SVA (cm) & $\mathrm{PI}\left({ }^{\circ}\right)$ & $\operatorname{LL}\left({ }^{\circ}\right)$ & $\begin{array}{c}\text { Potential Risk } \\
\text { Factors }\end{array}$ \\
\hline 1 & $52, F$ & Scoliosis & T9-L4 & L4-S1 & L-3 & 13 & 66 & -4 (kyphosis) & M \\
\hline 2 & $60, \mathrm{M}$ & Spinal stenosis & L1-S1 & T4-L4 & $L-5$ & 15 & 67 & 1 & S, M \\
\hline 3 & $63, F$ & DDD & L4-S1 & L2-L4 & $L-5$ & 13 & 59 & 15 & S, M \\
\hline 4 & $68, F$ & DDD & L4-S1 & T11-L4 & $L-5$ & 13 & 81 & 25 & $\mathrm{M}$ \\
\hline 5 & $68, F$ & Spondylolisthesis & L4-S1 & L1-L4 & $L-5$ & NA & 45 & -11 (kyphosis) & $\mathrm{S}, \mathrm{O}, \mathrm{M}$ \\
\hline 6 & $71, \mathrm{M}$ & Unknown & $\mathrm{T} 10-\mathrm{S} 1$ & $\begin{array}{l}\text { L3-4 for repair of } \\
\text { nonunion }\end{array}$ & $L-5$ & 13 & 61 & 5 & $\mathrm{~S}, \mathrm{O}, \mathrm{M}$ \\
\hline 7 & $74, \mathrm{~F}$ & Discitis & L2-S1 & T12-L5 & $L-5$ & NA & 67 & 58 & $\mathrm{O}, \mathrm{M}$ \\
\hline
\end{tabular}

$\mathrm{DDD}=$ degenerative disc disease; $\mathrm{M}=$ multiple previous surgeries; $\mathrm{NA}=$ not available (full-length standing radiographs); $\mathrm{O}=$ osteoporosis; $\mathrm{S}=$ smoker. 
attempt to restore overall sagittal alignment. For each patient, a solid fusion of the previously instrumented levels was confirmed both by preoperative CT and by intraoperative findings. The senior authors' standard protocol for revision spine surgery is hardware removal for complete exposure and investigation of the fusion mass. If solid fusion is noted, the hardware is generally not replaced at the fused segments. The adjacent segment was instrumented to the end of the fusion mass in each case.

\section{Fracture Description}

All 7 patients later developed a fracture within the fusion mass of the now uninstrumented segment. These fractures were spontaneous in all cases and occurred between 4 months and 4 years after hardware removal. In all cases, the patients presented with an increase in back pain that was chronic or subacute in nature and unrelated to any traumatic event. One patient had leg weakness, and 4 had progressive kyphosis.

The spinal level for fracture was L-5 in 6 of the 7 patients, which was the first uninstrumented level caudal to the newly placed hardware in all 6 of these patients. In the seventh patient, the fracture occurred at L-3, which was the adjacent proximal level to the new instrumentation. In all cases, the fracture was located in the posterolateral fusion mass. In 5 patients, the fracture also extended into at least 1 pedicle; in 2 patients, it additionally extended into the vertebral body (Fig. 1). SVA could be calculated for 5 of the 7 patients by using standing lateral scoliosis radiographs, with all patients having a sagittal imbalance of $13-$ $15 \mathrm{~cm}$ prior to development of the fracture. The patients with additional fractures had obvious sagittal imbalance; however, the imbalance could not be formally calculated due to the lack of scoliosis radiographs prior to fracture development. All patients had upright lumbar lateral radiographs, enabling calculation of PI as well as lumbar lordosis. All of the patients except 1 had significant PI/LL mismatch (Table 1).

\section{Surgical Outcomes}

Six patients with fracture underwent surgery due to the fracture of their fusion mass; 1 patient desired conservative treatment with bracing. Of those who underwent surgery, reinstrumentation was performed of the levels from which hardware was previously removed, along with anterior column support via anterior interbody fusion (1 patient) or direct lateral interbody fusion (2 patients). Pelvic fixation was performed in 1 patient. Pedicle subtraction osteotomy was required for correction of kyphosis in 4 patients. An attempt to restore overall sagittal balance was made in each of the 6 patients who underwent surgery after their fracture.

The 36 patients who had hardware removed, but did not sustain a fracture, formed a control group for analysis. In contrast to the patients sustaining a fracture, these patients had an average SVA of only $1.5 \mathrm{~cm}$. However, this patient population was somewhat different than the 7 patients who sustained a fracture. These patients tended to be younger (less than 50 years of age) and had hardware removed predominantly for pain, prominence, or infection, without symptomatic ASD or need for extension of fusion. Many were adult patients who had undergone surgery for adolescent idiopathic scoliosis. Only 7 of the 36 patients had hardware removed as part of an extension of fusion.

\section{Illustrative Case}

A 63-year-old woman (Case 3) with a history of smoking and osteoporosis presented with an acute increase in her back pain 5 months after surgery had been performed for ASD. Her index procedure had been an L-4 to sacrum fusion for spondylolisthesis, with the most recent surgery performed for symptomatic stenosis at L3-4 and L2-3, with progressive kyphosis at L1-2 and L2-3. An L2-4 laminectomy, L3-4 transforaminal interbody fusion, as well as an L1-4 posterolateral fusion was performed and, after intraoperative exploration of the L4-sacrum fusion demonstrated it to be solid, this hardware was removed and not replaced (Fig. 2). The patient's recovery was unremarkable, until she presented with new-onset back pain 5 months postoperatively. A CT myelogram was performed, demonstrating an L-5 compression fracture with resultant kyphosis (Fig. 2). A trial of bracing failed to resolve the patient's pain and the kyphosis progressed, prompting additional surgery. Her L1-4 fusion was extended to the pelvis.

\section{Discussion}

Many important factors surrounding spinal fusion continue to be elucidated. In particular, the indications, outcomes, and sequelae after hardware removal have not been studied extensively. Generally, hardware removal may be undertaken if the patient presents with hardware prominence or if the pain is relieved by hardware injection, or both, so long as a solid fusion has been achieved. In addition, removal of instrumentation is generally performed following percutaneous pedicle screw stabilization of
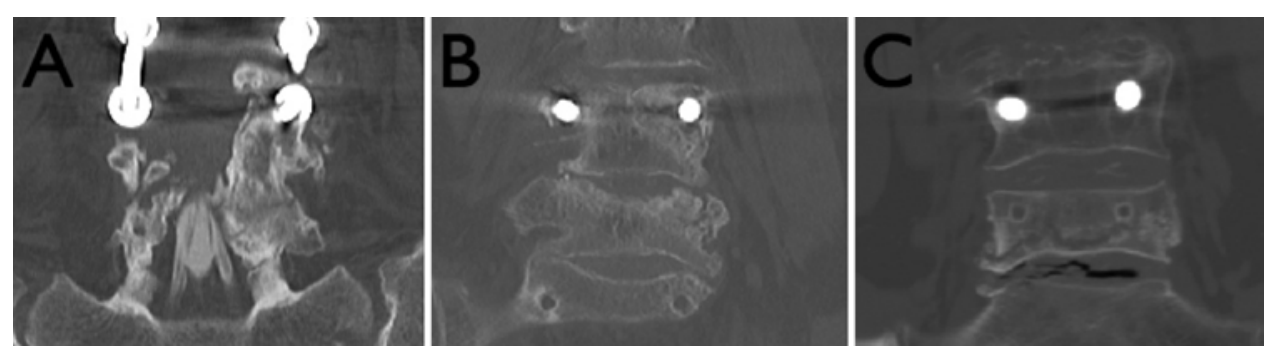

FIG. 1. Coronal CT scans demonstrating fracture of the posterolateral fusion mass (A), extension into the pedicle (B), and involvement of the vertebral body (C). 


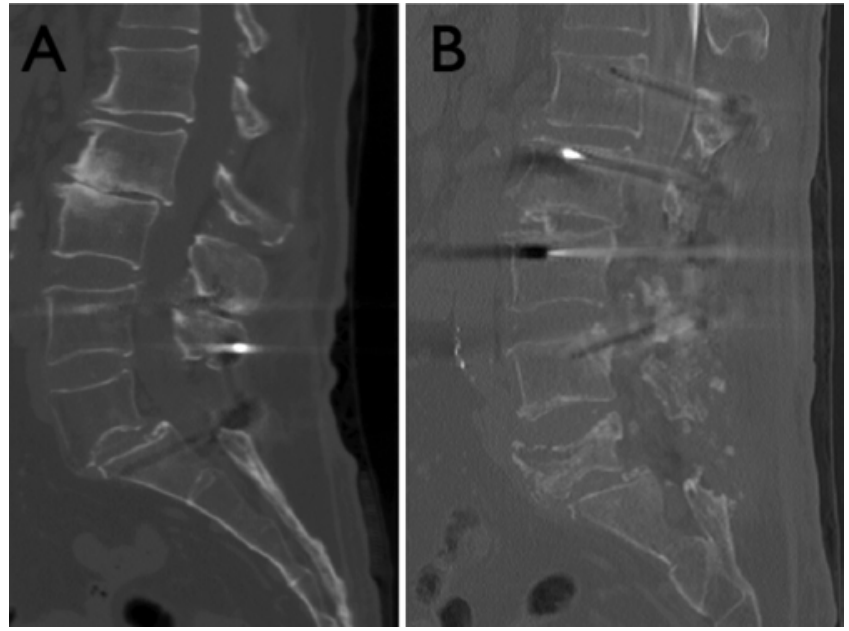

FIG. 2. Sagittal CT scans demonstrating preoperative anatomy prior to hardware removal $(\mathbf{A})$, and new fracture extending into the $L-5$ vertebral body after hardware removal (B).

fractures once bone healing has taken place. Additionally, if the patient requires surgery at adjacent levels, but demonstrates a mature fusion of the previously operated on levels, consideration may be given to removing all or part of the previous hardware. This treatment option has been reported in several case reports of patients with a wide range of problems and procedures. ${ }^{3-5,12}$

The sequelae of hardware removal are largely unknown, but have been studied best in the scoliosis literature. Potter and colleagues have reported on the loss of coronal correction following instrumentation removal for adolescent idiopathic scoliosis, but note the lack of change in the sagittal plane. ${ }^{9}$ Rathjen and colleagues also noted curve progression after hardware removal for idiopathic scoliosis in both the coronal and sagittal planes. ${ }^{11}$

The effects of screw-rod constructs on fusion mass strength have been studied. The effect of stress shielding on spinal instrumentation has been confirmed in a canine model. ${ }^{8}$ This study may be pertinent in our case series, particularly given the remote initial surgery for some patients, and the likely use of stainless steel rods in their index procedure, which have a higher modulus of elasticity than the currently used cobalt-chrome or titanium rods, thereby increasing the stress shielding effect.

All of the fractures in our series were adjacent to the new instrumentation. Spontaneous fracture of levels adjacent to fusion has been previously reported by Yasuhara and colleagues, who describe 2 cases of spontaneous L-1 fracture after 4-level fusions for lumbar degenerative disease in osteoporotic patients, ${ }^{14}$ although in these patients, the fractured level was a single, nonfused segment. Similarly, Watanabe and colleagues described a series of 10 patients with a proximal junctional fracture of an unfused level after spinal deformity surgery. Risk factors in their series included advanced age, osteopenia, preoperative comorbidities, and severe global sagittal imbalance. ${ }^{13}$ In our series, only 3 patients had confirmed osteoporosis; however, 5 had severe sagittal imbalance (SVA of at least $13 \mathrm{~cm}$ ), and the additional patients also had sagittal imbalance, although it could not be calculated. Furthermore, all patients except 1 had PI/LL mismatch on upright lumbar imaging prior to fracture. In contrast, the patients who underwent hardware removal but experienced no fracture of their fusion mass had overall better sagittal balance. There was no patient with a fracture who had an SVA less than $13 \mathrm{~cm}$; conversely, no patient without a fracture had an SVA greater than $9 \mathrm{~cm}$, with most having adequate sagittal balance and an SVA less than $5 \mathrm{~cm}$.

All of the patients sustaining a fracture in the present series had hardware removed during a surgery for adjacent level disease, often due to proximal junctional kyphosis. Radcliff and colleagues have demonstrated a rate of ASD to be approximately $2 \%-3 \%$ per year after a variety of decompression and stabilization procedures, with sagittal imbalance as a risk factor..$^{10}$ In a recent article by Lee and colleagues, statistically significant risk factors for proximal junctional kyphosis included advanced age, upper instrumented vertebra below L-2, lumbar lordosis to pelvic incidence ratio, and the sum of lumbar lordosis and sacral slope related to pelvic incidence. ${ }^{7}$ Although a retrospective review by Glattes and colleagues was unable to establish sagittal imbalance as a risk factor for proximal junctional kyphosis, ${ }^{2}$ a systematic review of 7 retrospective cohorts studying patients with long thoracolumbar fusions found a weak association of distal ASD with a higher preoperative sagittal imbalance. ${ }^{6}$

Sagittal imbalance has previously been related to outcome, with Berjano and colleagues suggesting in a report of 12 patients that inadequate restoration of sagittal balance increases risk of failures and revisions. ${ }^{1}$ Although in the current study we cannot establish a causative effect, all of the patients in our series who sustained a spontaneous fracture of the fusion mass underwent hardware removal during a surgery for ASD and all had severe sagittal imbalance, suggesting that complete hardware removal should be avoided in such cases. In contrast, patients with hardware removal who had better overall sagittal balance did not experience this complication. The overall occurrence of this phenomenon in our patient series was $16 \%$, but all of those patients had an SVA greater than $10 \mathrm{~cm}$. The development of a spontaneous fracture of a solid fusion mass may be related to positive sagittal imbalance. When performing revision fusions for adjacent level pathology, the primary goal should always be to restore proper sagittal alignment; however, in cases when this is impossible due to patient comorbidities or their desire for a smaller surgery, consideration should be given to reimplanting hardware across solid fusions to prevent spontaneous fracture of these areas.

\section{Conclusions}

We present a case series of 7 patients who underwent surgery for ASD after a remote fusion who later developed a fracture of the fusion mass after hardware removal from their previously successfully fused segment. This complication may be related to sagittal imbalance, and consideration should be given to reimplanting hardware for these patients, even across good fusions, to prevent spontaneous fracture of these areas if the sagittal imbalance is not corrected. 


\section{Acknowledgments}

We wish to acknowledge Nina Clovis, Suzanne Danley, and Sheila Rye for their assistance with the preparation of this paper. No funding was received for this study.

\section{References}

1. Berjano P, Bassani R, Casero G, Sinigaglia A, Cecchinato R, Lamartina $\mathrm{C}$ : Failures and revisions in surgery for sagittal imbalance: analysis of factors influencing failure. Eur Spine J 22 (Suppl 6):S853-S858, 2013

2. Glattes RC, Bridwell KH, Lenke LG, Kim YJ, Rinella A, Edwards C II: Proximal junctional kyphosis in adult spinal deformity following long instrumented posterior spinal fusion: incidence, outcomes, and risk factor analysis. Spine (Phila Pa 1976) 30:1643-1649, 2005

3. Ha KY, Kim YH: Bilateral pedicle stress fracture after instrumented posterolateral lumbar fusion: a case report. Spine (Phila Pa 1976) 28:E158-E160, 2003

4. Ha KY, Kwon SE, Kim KW, Oh IS, Lee YM: Vertebral compression fracture in the middle of fused segments without a history of injury: a case report. Spine (Phila Pa 1976) 35:E137-E139, 2010

5. Ito T, Endo N, Honma T, Hirano T: Late stress fracture of a well-consolidated strut graft after total spondylectomy in the thoracolumbar spine. Spine (Phila Pa 1976) 36:E551-E555, 2011

6. Kasliwal MK, Shaffrey CI, Lenke LG, Dettori JR, Ely CG, Smith JS: Frequency, risk factors, and treatment of distal adjacent segment pathology after long thoracolumbar fusion: a systematic review. Spine (Phila Pa 1976) 37 (22 Suppl):S165-S179, 2012

7. Lee JH, Kim JU, Jang JS, Lee SH: Analysis of the incidence and risk factors for the progression of proximal junctional kyphosis following surgical treatment for lumbar degenerative kyphosis: minimum 2-year follow-up. Br J Neurosurg 28:252-258, 2014

8. McAfee PC, Farey ID, Sutterlin CE, Gurr KR, Warden KE, Cunningham BW: The effect of spinal implant rigidity on vertebral bone density. A canine model. Spine (Phila Pa 1976) 16 (6 Suppl):S190-S197, 1991

9. Potter BK, Kirk KL, Shah SA, Kuklo TR: Loss of coronal correction following instrumentation removal in adolescent idiopathic scoliosis. Spine (Phila Pa 1976) 31:67-72, 2006
10. Radcliff KE, Kepler CK, Jakoi A, Sidhu GS, Rihn J, Vaccaro AR, et al: Adjacent segment disease in the lumbar spine following different treatment interventions. Spine J 13:13391349,2013

11. Rathjen K, Wood M, McClung A, Vest Z: Clinical and radiographic results after implant removal in idiopathic scoliosis. Spine (Phila Pa 1976) 32:2184-2188, 2007

12. Waelchli B, Min K, Cathrein P, Boos N: Vertebral body compression fracture after removal of pedicle screws: a report of two cases. Eur Spine J 11:504-506, 2002

13. Watanabe K, Lenke LG, Bridwell KH, Yongjuing KJ, Loester L, Hensley M: Proximal junctional vertebral fracture in adults after spinal deformity surgery using pedicle screw constructs: analysis of morphological features. Spine (Phila Pa 1976) 35:138-145, 2010

14. Yasuhara T, Takahashi Y, Kumamoto S, Nakahara M, Yoneda K, Niimura T, et al: Proximal vertebral body fracture after 4-level fusion using 11 as the upper instrumented vertebra for lumbar degenerative disease: report of 2 cases with literature review. Acta Med Okayama 67:197-202, 2013

\section{Disclosures}

Scott D. Daffner has stock in Pfizer and Amgen and has had speaking/travel support from DePuy-Synthes. He is a co-investigator on a grant from the Cervical Spine Research Society. Sanford E. Emery has an endowment from the State of West Virginia Research Endowment and is the principal investigator on a grant from the Cervical Spine Research Society. John C. France is a co-investigator on a grant from the Cervical Spine Research Society.

\section{Author Contributions}

Conception and design: all authors. Acquisition of data: all authors. Analysis and interpretation of data: all authors. Drafting the article: Sedney, Daffner, Stefanko, Emery, France. Critically revising the article: Sedney, Daffner, Emery, France. Reviewed submitted version of manuscript: all authors. Approved the final version of the manuscript on behalf of all authors: Sedney.

\section{Correspondence}

Cara L. Sedney, Department of Neurosurgery, West Virginia University, P.O. Box 9183, Morgantown, WV 26506-9183. email: csedney@hsc.wvu.edu. 